\title{
Recurrent Optic Neuritis and Acute Encephalopathy with Myelin Oligodendrocyte Glycoprotein Antibodies in a Korean Child
}

\author{
Ji Eun Hwang', Su-Hyun Kim², Soyoung Park' \\ 'Department of Pediatrics, Soonchunhyang University Bucheon Hospital, Bucheon; ${ }^{2}$ Department of Neurology, Research Institute and Hospital, National \\ Cancer Center, Goyang, Korea
}

\begin{abstract}
Myelin oligodendrocyte glycoprotein (MOG) antibody-associated diseases are reported to have distinct pathogenic mechanisms, clinical courses, and therapeutic implications in comparison to other acquired demyelinating syndromes. Here, we report the case of a 6-year-old Korean girl with recurrent steroid-responsive optic neuritis (ON) and an episode of acute disseminated encephalomyelitis (ADEM) with a high MOG antibody titer. Initially, she suffered from left ON with subclinical brain lesions including magnetic resonance imaging hyperintensities in the bilateral frontal subcortical white matter, the left thalamus, and the right cerebral peduncle. Her vision improved dramatically after steroid therapy; however, 3 months after the 1st episode, she developed acute encephalopathy with extensive newly developed ADEM-like lesions and resolution of previous hyperintensities. Despite regular interferon $\beta$-1a injection after steroid tapering, she developed a 3rd episode presenting as a right ON. Throughout these three episodes, serological tests associated with autoimmune diseases, viral markers, anti-aquaporin-4 antibodies, and cerebrospinal fluid oligoclonal bands were negative, with the immunoglobulin $\mathrm{G}$ index in the normal range. After the 3rd episode, anti-MOG antibody testing was performed and higher titer was detected. Testing for anti-MOG antibodies is highly recommended for relapsing inflammatory demyelinating central nervous system diseases in pediatric patients, in order to define early an adequate long-term treatment.
\end{abstract}

Keywords: Brain diseases; Child; Myelin-oligodendrocyte glycoprotein; Optic neuritis

\section{INTRODUCTION}

Inflammatory demyelinating central nervous system (CNS) diseases as diverse as neuromyelitis optica (NMO), multiple sclerosis (MS), transverse myelitis, acute disseminated encephalomyelitis (ADEM), or optic neuritis (ON) have shared clinical, radiological, and immunological features, particularly at onset $[1,2]$. Many demyelinating disease-associated antibodies have been described [3,4] as important diagnostic and prognostic biomarkers. Recently, antibodies against myelin oligodendrocyte glycoprotein (MOG) have gained attention. High titers of anti-MOG antibodies are reported to be predominant in pediatric patients with acquired inflammatory demyelinating diseases of the CNS such as recurrent ONs, ADEM followed by ON, and multiphasic disseminated encephalomyelitis [2,5-7]. Patients with anti-MOG antibodies appear to have a unique clinical, radiological, and therapeutic pro- file. Therefore, new specific therapeutic strategies for anti-MOG antibody-associated disease are under development. Because few cases of MOG seropositivity have been reported in Asian children $[8,9]$, we here describe a Korean child with recurrent unilateral ONs and a single ADEM episode who showed high titers of MOG antibodies. This study was approved by the institutional review board of the Soonchunhyang University Bucheon Hospital with informed consent (2019-11-041).

\section{CASE REPORT}

A 6-year-old Korean girl with sudden loss of vision in her left eye visited Soonchunhyang University Bucheon Hospital. She had been healthy previously and had an unremarkable medical and family history. The ophthalmologic examination revealed a decreased visual acuity, loss of color vision, and the fundoscopy
Correspondence to: Soyoung Park

Department of Pediatrics, Soonchunhyang University Bucheon Hospital, 170 Jomaru-ro, Wonmi-gu, Bucheon 14584, Korea

Tel: +82-32-621-5403, Fax: +82-32-621-5018, E-mail: hclove72@schmc.ac.kr

Received: Oct. 21, 2019 / Accepted after revision: Dec. 3, 2019
(C) 2019 Soonchunhyang Medical Research Institute

This is an Open Access article distributed under the terms of the Creative Commons Attribution Non-Commercial License (http://creativecommons.org/licenses/by-nc/4.0/). 
showed a left optic disc swelling. Brain and orbit magnetic resonance imaging (MRI) revealed enlarged left optic nerve with perineural fat infiltration on T2-weighted images (T2-WIs) (Fig. 1A), as well as increased signal intensities on T2-WIs and fluid-attenuated inversion recovery images in the bilateral frontal subcortical white matter, left thalamus, and right cerebral peduncle; notably, there were no neurological symptoms (Fig. 1B). The lesions had subtle signal changes in diffusion images and were not enhanced with gadolinium. Her cerebrospinal fluid (CSF) showed lymphocytosis, but protein and sugar were in the normal range (Table 1). No organisms were found in her serum or CSF; oligoclonal bands (OCBs) and anti-aquaporin-4 antibodies (AQP4 abs) were negative. After high-dose intravenous (IV) methylprednisolone for 5 days followed by a tapering course of oral steroids for 8 weeks, her visual acuity improved dramatically from finger counting only to 0.6 after 1 month after the 1st episode. Maintenance treatment for acquired demyelinating syndrome was not started at that time; however, due to this clinically isolated syndrome with subclinical brain MRI lesions, she visited the outpatient clinic regularly under the strong suspicion of a relapsing course of an acquired demyelinating disease. One month after cessation of the oral steroids, she complained of drowsiness, poor appetite, and gait disturbances with mild fever for several days. On her second brain MRI, she presented newly developed, extensive, and ill-defined hyperintensities on T2-WIs. These lesions were detected in the subcortical white matter around the 4 th ventricle, deep gray matter, both mul-
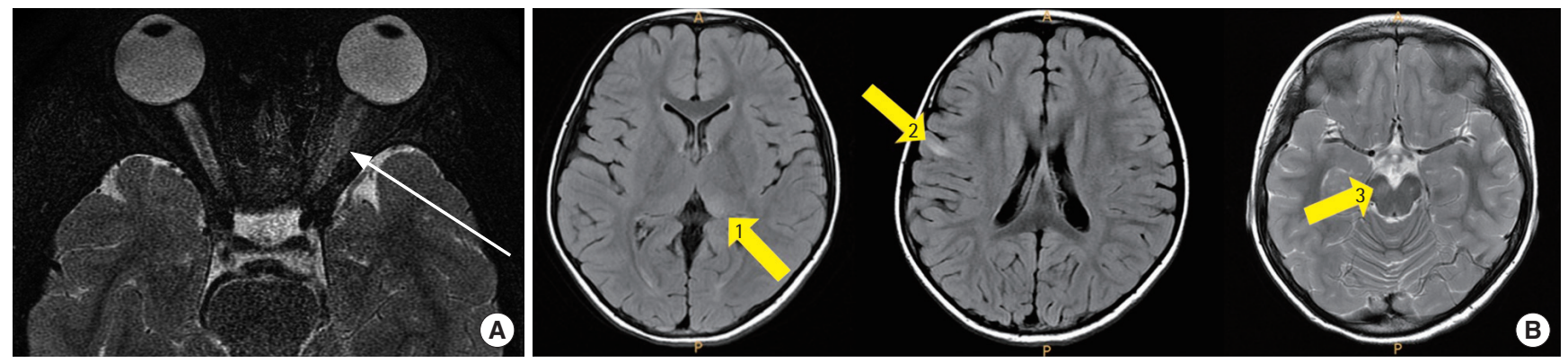

Fig. 1. Orbital and brain magnetic resonance imaging during the 1st episode presenting as a left-sided optic neuritis with subclinical abnormalities. (A) Enlarged left optic nerve with infiltration of the perineural fat on T2WI (arrow). (B) Hyperintensities on T2 FLAIR image in the left thalamus (yellow arrow 1). Hyperintensities on T2 FLAIR image in right frontal subcortex (yellow arrow 2). Hyperintensities on T2WI image on right peduncle (yellow arrow 3). T2WI, T2 weighted image; FLAIR, fluid-attenuated inversion recovery.

Table 1. CSF and serologic studies for each of three demyelinating episodes in this patient

\begin{tabular}{|c|c|c|c|}
\hline Characteristic & 1st episode & 2nd episode & 3rd episode \\
\hline \multicolumn{4}{|l|}{ CSF study } \\
\hline Immunoglobulin G index (normal <0.7) & 0.0007 & 0.0006 & 0.0008 \\
\hline CSF oligoclonal band & Negative & Negative & Negative \\
\hline CSF cell count (polymorphonucleocytes \%) & $24(45)$ & $22(41)$ & 0 \\
\hline CSF protein & 24.8 & 47.1 & 18.8 \\
\hline Herpes simplex virus type I \& II, varicella-zoster virus, Epstein-Barr virus, enterovirus PCR & All negative & All negative & All negative \\
\hline Mycoplasma PCR & Negative & Negative & Negative \\
\hline Fungal/bacterial culture & All negative & All negative & All negative \\
\hline \multicolumn{4}{|l|}{ Serologic study } \\
\hline Serum anti-aquaporin-4 antibody & Negative & Negative & Negative \\
\hline $\begin{array}{l}\text { Antibodies associated with coexisting other autoimmune disorders (i.e., systemic lupus } \\
\text { erythematosus or Sjögren syndrome) }\end{array}$ & All negative & All negative & All negative \\
\hline Antinuclear antibody & Negative & Negative & Negative \\
\hline Erythrocyte sedimentation rate, C-reactive protein & All negative & All negative & All negative \\
\hline Human leukocyte antigen-DR beta 15 & - & Negative & - \\
\hline Thyroid-stimulating hormone & - & Within normal range & Within normal range \\
\hline Vitamin D & - & Low & Low \\
\hline
\end{tabular}

CSF, cerebrospinal fluid; PCR, polymerase chain reaction. 
tifocal cortexes, left cerebral peduncle, and right thalamus. Neither the previous hyperintense lesions nor a swelling of the optic nerve were detectable (Fig. 2). Additionally, spinal MRI T2 images showed newly apparent ill-defined hyperintense lesions at the C3 level. After steroid pulse therapy, she nearly fully recovered within 3 days. The clinical symptoms, the brain MRI, and the CSF tests satisfied the diagnostic criteria for ADEM; considering her previous episode of steroid-responsive unilateral ON, regular subcutaneous injections of interferon (IFN) $\beta$-1a were started, accompa- nied by oral steroid tapering. At the 2 nd episode her visual acuity improved to 0.9 on the left side.

Despite regular maintenance treatment with IFN $\beta$-1a, she experienced a 3rd episode, 3 months later, as a unilateral right ON. This time, the brain and spinal MRIs showed no newly developed lesion, and the extensive previous lesions had disappeared. After high-dose steroid injection for 5 days, the visual acuity of the right eye improved gradually from 0.2 to 0.6 . Throughout these three episodes, serological tests associated with autoimmune diseases,
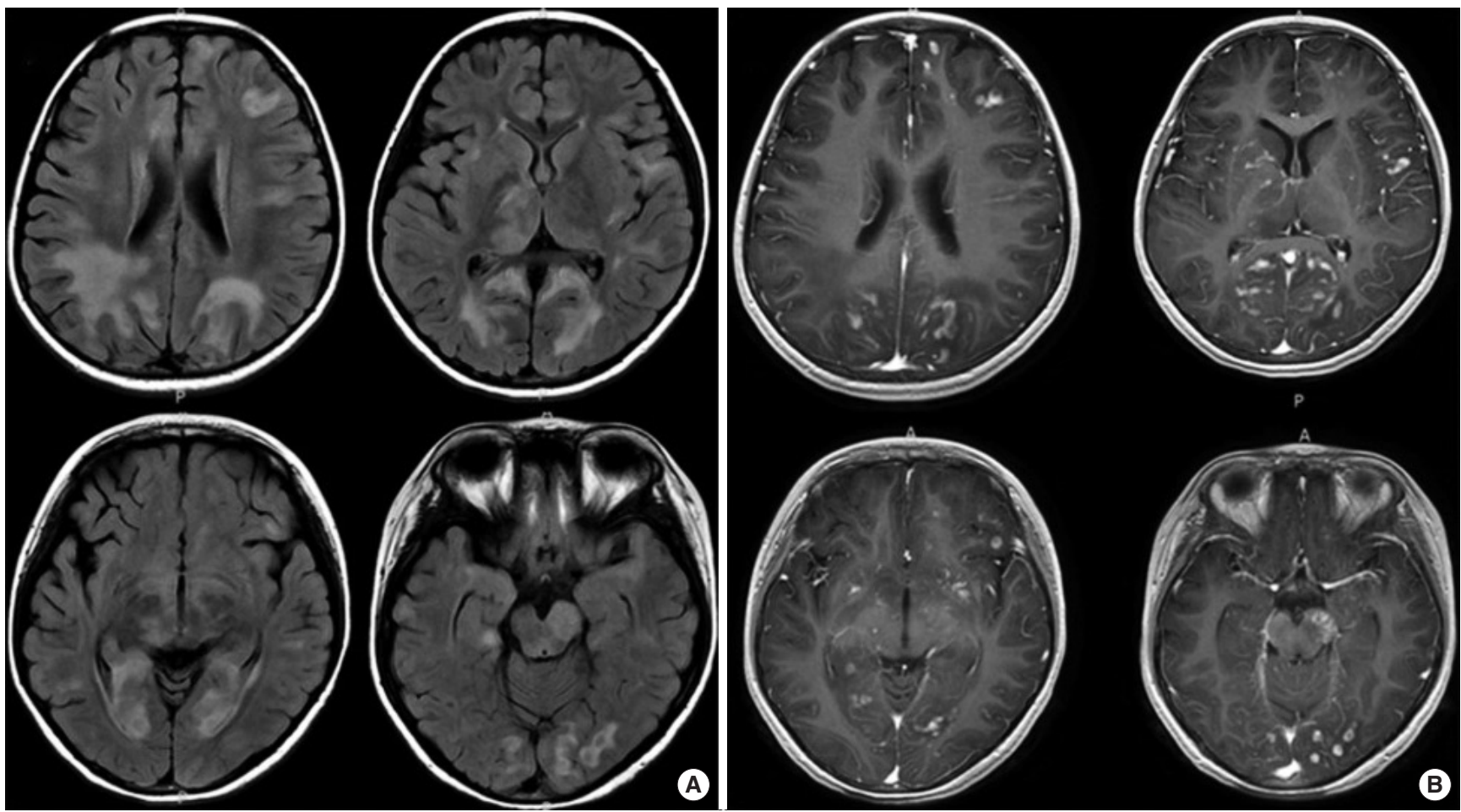

Fig. 2. (A) Brain MRI during the 2nd episode presenting as acute disseminated encephalomyelitis: extensive ill-defined hyperintense lesions in T2 fluid-attenuated inversion recovery images in the subcortical white matter, the deep gray matter, the left cerebral peduncle, and the right thalamus with resolution of the previous lesions. (B) Brain MRI during the 2nd episode on T1-enhanced images in the same areas. MRI, magnetic resonance imaging.
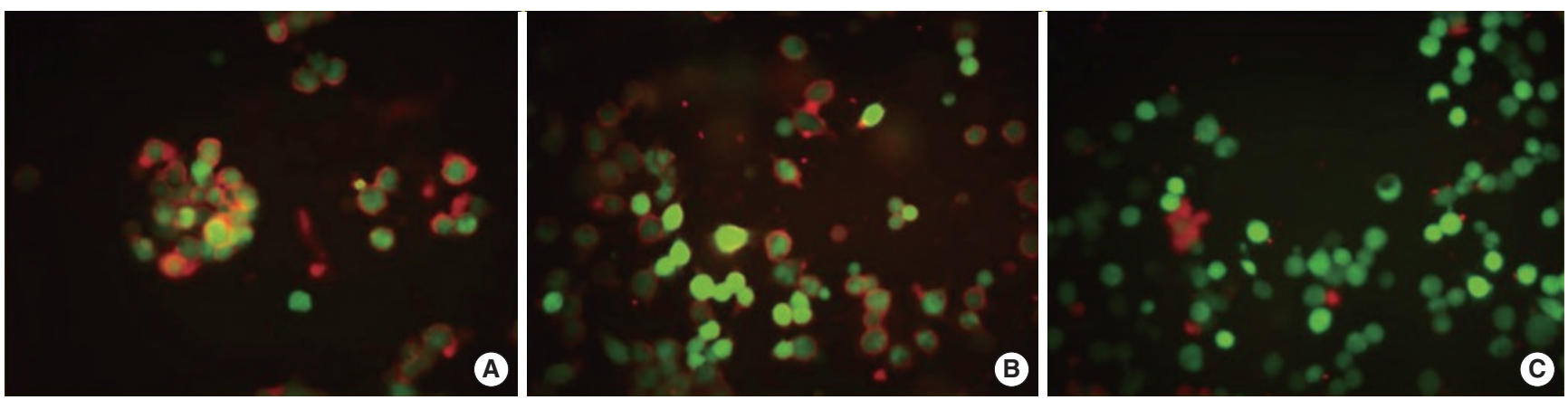

Fig. 3. Serological test for anti-MOG antibody in this patient with a standardized cell-based assay using human embryonic kidney 293 cells and MOG-immunoglobulin G. (A) Positive control, (B) patient's serum, and (C) negative control. MOG, myelin oligodendrocyte glycoprotein. 
viral markers, anti-AQP4 abs, and CSF OCBs were all negative, with the immunoglobulin $\mathrm{G}$ index in the normal range (Table 1). Finally, a serological examination for anti-MOG antibodies was performed, and a high titer of serum anti-MOG antibody was detected (Fig. 3). As a long-term treatment of MOG autoantibodyassociated demyelinating diseases, regular IV immunoglobulin or mycophenolate mofetil was considered as a treatment option in this patient.

\section{DISCUSSION}

In this patient, we decided to test anti-MOG antibody considering the following facts: recurrent unilateral $\mathrm{ON}$ at a young age, mainly with ADEM-like and deep gray matter lesions in the brain MRI; good response to steroids; relapse despite IFN $\beta$-1a treatment; and anti-AQP4 ab-negative findings in serologic analysis. Recently, MOG antibodies have been extensively studied in relapsing courses of demyelinating syndromes, especially in pediatric cases. They sometimes meet the existing MS and NMO spectrum disorder (NMOSD) criteria, but exhibit different clinical courses, which is reflected in the revised criteria [10]. The treatment strategy for MOG antibody-positive diseases has not yet been fully established, and the evidence for an optimal approach is undergoing collection. IV methylprednisolone is considered an effective drug in the acute phase and IV immunoglobulin was reported to be helpful in prevention of relapse [6]. Corticosteroids, mycophenolate mofetil, and methotrexate have all been used as disease-modifying drugs, while immunomodulating drugs for MS, such as IFN, have typically been ineffective $[1,6]$. We recommend performing a MOG antibody test in children who exhibit suspected atypical MS or relapsing anti-AQP4 ab-negative NMOSD. This establishes the correct diagnosis and enables proper long-term treatment.

\section{ACKNOWLEDGMENTS}

This work was supported by Soonchunhyang University Research Fund. We are grateful to Dr. Ho Jin Kim, M.D., Ph.D. (Department of Neurology, Research Institute, National Cancer Center, Goyang, Korea) for anti-AQP4 and anti-MOG antibody analyses and also to Dr. Jee Ho Chang, M.D., Ph.D. (Department of Ophthalmology, Soonchunhyang University Bucheon Hospital, Soonchunhyang University College of Medicine, Bucheon, Korea) for assessment of ophthalmologic status.

\section{REFERENCES}

1. Reindl M, Di Pauli F, Rostasy K, Berger T. The spectrum of MOG autoantibody-associated demyelinating diseases. Nat Rev Neurol 2013;9:455-61.

2. Hennes EM, Baumann M, Schanda K, Anlar B, Bajer-Kornek B, Blaschek A, et al. Prognostic relevance of MOG antibodies in children with an acquired demyelinating syndrome. Neurology 2017;89:900-8.

3. Armangue T, Yeshokumar AK, Sepulveda M, Graus F, Saiz A. Antibodies in acquired demyelinating disorders in children. Mult Scler Demyelinating Disord 2016;1:5.

4. Jarius S, Paul F, Aktas O, Asgari N, Dale RC, de Seze J, et al. MOG encephalomyelitis: international recommendations on diagnosis and antibody testing. J Neuroinflammation 2018;15:134.

5. Lechner C, Baumann M, Hennes EM, Schanda K, Marquard K, Karenfort $\mathrm{M}$, et al. Antibodies to MOG and AQP4 in children with neuromyelitis optica and limited forms of the disease. J Neurol Neurosurg Psychiatry 2016;87:897-905

6. Hacohen Y, Wong YY, Lechner C, Jurynczyk M, Wright S, Konuskan B, et al. Disease course and treatment responses in children with relapsing myelin oligodendrocyte glycoprotein antibody-associated disease. JAMA Neurol 2018;75:478-87.

7. Ramanathan S, Mohammad S, Tantsis E, Nguyen TK, Merheb V, Fung VS, et al. Clinical course, therapeutic responses and outcomes in relapsing MOG antibody-associated demyelination. J Neurol Neurosurg Psychiatry 2018;89:127-37.

8. Tsuburaya RS, Miki N, Tanaka K, Kageyama T, Irahara K, Mukaida S, et al. Anti-myelin oligodendrocyte glycoprotein (MOG) antibodies in a Japanese boy with recurrent optic neuritis. Brain Dev 2015;37:145-8.

9. Nishiyama M, Nagase H, Matsumoto M, Tomioka K, Awano H, Tanaka T, et al. Clinical features in very early-onset demyelinating disease with antiMOG antibody. Brain Dev 2017;39:756-62.

10. Thompson AJ, Banwell BL, Barkhof F, Carroll WM, Coetzee T, Comi G, et al. Diagnosis of multiple sclerosis: 2017 revisions of the McDonald criteria. Lancet Neurol 2018;17:162-73. 